\title{
Identification of Existing Agroforestry Systems and Socio-Economic Assessment in Kandaghat Block of Solan District, Himachal Pradesh, India
}

\author{
Pradeep Kumar $^{1 *}$, C.L. Thakur ${ }^{1}$, Pradeepen Rai ${ }^{1}$ and Kaushal Attri ${ }^{2}$ \\ ${ }^{1}$ Department of Silviculture and Agroforestry, ${ }^{2}$ Department of Plant Pathology, Dr. Yashwant \\ Singh Parmar University of Horticulture and Forestry, Nauni 173230, India \\ *Corresponding author
}

\begin{tabular}{|l|}
\hline K e y w o r d s \\
$\begin{array}{l}\text { Agroforestry system, } \\
\text { Farmers' category, } \\
\text { Socio-economic, Sex- } \\
\text { ratio }\end{array}$ \\
\hline Article Info \\
\hline $\begin{array}{l}\text { Accepted: } \\
\text { 30 March } 2018 \\
\text { Available Online: } \\
\text { 10 April 2018 }\end{array}$ \\
\hline
\end{tabular}

\section{Keywords}

Socio-economic, Sex

ratio

ticle Info

30 March 2018

10 April 2018

\section{A B S T R A C T}

The study was carried out at Kandaghat block in Solan district of Himachal Pradesh with the aim to identify different existing agroforestry systems and to assess the demographic and socio-economic conditions of farmers. Agrisilviculture (AS), Agrisilvipastoral (ASP), Agrisilivihorticulture (ASH), Agrihortisilviculture (AHS), Hortipastoral (HP) and Silvipastoral (SP) were prominent system in the studied area. Five types of agroforestry systems were practised by the marginal and six systems each by small and medium categories. Amongst them, ASP and SP were most widely adopted irrespective of farmers' category. Average family members per household was in the order: Medium (6.8) > Small (6.2) > Marginal (6.1). Sex ratio was observed as Small (1138) > Marginal (1033) > Medium category (943). Males dominated over women in literacy as well as in all the income generation avenues except in the farm labor. Cow was the most preferred domesticated animal followed by buffaloes, goats and sheep. Average area under arable land constitutes 53.68 per cent combining both irrigated and unirrigated areas, out of which major portion of arable land is dependent on rainfall. Besides, considerable amount of arable land was also under irrigation facilities. The system units in agriculture components were wheat, pea, mustard, barley and cabbage, cauliflower as rabi crops, whereas maize, colocassia, beans, capsicum, tomato, ginger and turmeric as kharif crops. Tree components were Grewia optiva, Celtis australis, Pinus roxburghii, Robinia pseudoacasia, Quercus leucotrichophora, Bauhinia variegata, Myrica esculanta, Pistacia integerrima, Toona ciliata, Pyrus pashia, Morus alba, Ficus roxburghii, Ficus palmata and Prunus padus which were retained on the bunds of agriculture fields, whereas fruit trees of Prunus armeniaca, Pyrus communis and Prunus saliciana were planted in and around the agriculture fields. The dominant grass species were observed as Chrysopogon montanus, Heteropogon contortus, Panicum maximum, Apluda mutica, Dicanthium annulatum, Themada anathera, Ischaemum aristatum, Arundinella nepalensis and Oplismenus compositus.

\section{Introduction}

Agroforestry refers to practices, which deliberately or intentionally mix or maintain woody perennials on the crop/animal production systems. It combines essentials of agriculture, crops and/ or animals with elements of forestry in production system in a 
unit of land, either simultaneously or sequentially. Agroforestry systems are socially accepted by mountain communities as among the most effective practices for conserving soil, water, and improving fertility, while at the same time providing a valuable source for food, timber, fodder and many other nontimber forest products (NTFP). Mercer (1993) states that agroforestry projects have two main objectives: first, to increase the efficiency of the use of rural resources by reducing or eliminating ecologically destructive land-use practices and by introducing new or improved agroforestry enterprises in order to produce sustainable increases in incomes and living standards, and second, to provide for social equity. Estimating the socio-economic impact of an agroforestry system is a way to measure the success of meeting the above fundamental objectives of economic and social equity/distributive efficiency. The ecosystem services provided by agroforestry are noteworthy for their positive contributions towards the water, carbon, and nitrogen cycles, as well as for their cultural and socioeconomic potentials in precipitous mountainous regions. In the hills, the agriculture production is supported by the inputs from the forest in the form of fuel wood, fodder and manure. The variation in the ecosystem services is explained by social factors such as education level, income level and gender. (Al-Assaf et al., 2014, Hossai et al., 2016) The prosperity of the hill communities has traditionally being dependent on the forest, farming, agroforestry and livestock. The main cause of the poor economic status is degradation of the forest ecosystem, less farm production, poor watershed management and lack of infrastructure development. Social factors such as farmers, economic and educational status, demography, social connection, and culture and resource availability are important to understand why and how farmers select certain farming practices (Seabrook et al.,
2008.) In the rural areas people for their livelihood exclusively dependent on the forest, farming including agroforestry and livestock or migrate to the plains in search of employment. Comparative studies on agroforestry systems are however, still lacking in the Himachal Pradesh. Hence, the present study was undertaken to assess socioeconomic aspect of existing agroforestry systems in sub temperate region of Himachal Pradesh.

\section{Materials and Methods}

The study was carried out during the year 2015-16 at Kandaghat block of Solan district in Himachal Pradesh with geographical coordinates between $33^{0} 30^{\prime}$ to $31^{0} 15^{\prime} \mathrm{N}$ latitude and $76^{\circ} 42^{\prime}$ to $77^{\circ} 20^{\prime} \mathrm{E}$ longitude. The block comes under sub-temperate to subhumid mid hill zone. Study sites were selected through stratified multi-stage random sampling in which two panchayats i.e. Kwarag and Sirinagar panchayats were chosen and each panchayat was further divided into two villages. In each village, farmers were divided into three different categories (Marginal <1ha, Small 1-2ha and Medium 2-5 ha) on the basis of their land holding as per the classification of government of Himachal Pradesh. Fifteen farmers were selected from each village, which includes five farmers each from marginal, small and medium category. Estimate of the family structure, demographic feature, livestock status, animal husbandry practices, land utilization pattern, tree inventory and crop production from various agroforestry systems was met through sets of tabular questionnaires and field visits.

\section{Results and Discussion}

\section{Identification of Agroforestry systems types}

In total six agroforestry systems were identified in the study area (Table 1). 
However, five agroforestry systems were identified in marginal category and six each in small and medium categories of farmers. These systems are enlisted as: Agrisilviculture (AS), Agrisilvipastoral (ASP), Agrisilivi horticulture (ASH), Agrihortisilviculture (AHS), Hortipastoral (HP) and Silvipastoral (SP). It is evident from the table that Silvipastoral (SP) system dominated in all the existing agroforestry systems in different farmers' categories. This system was found to be practiced by 33.08 per cent of the sampled farmers and the least practised system in vogue among them was Hortiipastoral (HP) contributing only 4.51 per cent. The second most important agroforestry system observed was Agrisilvipastoral (ASP) with 27.82 per cent followed by Agrisilvihorticulture (ASH), Hortipastoral (HP) on overall basis. In marginal category of farmers Hortipastoral system was found to be absent and which can be due to the reason of less availability of the land for further diversification of other agroforestry systems. Silvipastoral (SP) system was observed maximum for medium farmer and small category. The least preferred system for small and medium category of farmers was Hortipastoral (HP). Thus, it can be concluded that only two systems i.e. Silvipastoral (SP) system and Agrisilvipastoral (ASP) were most prevalen among all the sampled farmers' categories.

The functional units under agricultural component were cereals e.g. wheat, maize and barley; oilseeds and pulses- mustard; Vegetables- Cauliflower, cabbage, colocassia, beans, capsicum, tomato, pea, turmeric. The silviculture component was represented by Grewia optiva, Celtis australis, Pinus roxburghii, Robinia pseudoacasia, Quercus leucotrichophora, Bauhinia variegata, Myrica esculanta, Pistacia integerrima, Toona ciliata, Pyrus pashia, Morus alba, Ficus roxburghii, Ficus palmata and Prunus padus. Major fruits tree species includes Prunus armeniaca, Pyrus communis and Prunus saliciana while major grasses species present were Chrysopogon montanus, Heteropogon contortus, Panicum maximum, Apluda mutica, Dicanthium annulatum, Themada anathera, Ischaemum aristatum, Arundinella nepalensis and Oplismenus compositus. Different combinations of trees, agri-crops and grasses species were recorded in different AFS which varied among farmers' category. Similar agroforestry systems have been identified and reported by many workers in Himachal Pradesh. Upadhyaya (1997) reported six agroforestry systems namely, agrisiliviculture, agrisilvihorticulture, pastoralsilvihorticultural, pastoralhortisilviculture and silvipasture in Balh valley of Himachal Pradesh. Likewise, Kachru (1997) reported eight agroforestry systems namely, agrisilviculture (Maize, Wheat, Blackgram and Grewia, Morus), agrihorticulture (Wheat, Mustard and Pear), agrisilvihorticulture (Wheat, Mustard, Grewia and Apricot), pastoralsilviculture (Grewia, Bauhinia and grasses), pastoralhortisilviculture (Pear, Plum, Grewia and grasses), agrihortisilviculture (Maize, Blackgram Pear and Grewia), pastoralsilviculture (Chir pine, Acacia and grasses), and pasture in mid-hills of Himachal Pradesh. Kumari et al., (2008) reported five agroforestry systems in Lahaul and Kinnuar in Himachal Pradesh viz., agrihorticulture (Pea, Potato and Apple), agrisilviculture (Pea, Potato, Rajmah and Salix), agrisilvipastoral (Pea, Salix and grasses), pastoralsilviculture (grasses and Salix) and pastoralhorticulture (grasses and Apple). Goswami (2009) reported five agroforestry systems viz., agrisilvihorticulture (Maize, Wheat, Blackgram, Grewia, Ficus and Pear), agrohortisilviculture (Wheat, Rajmah, Tomato, Pomegranate and Grewia), agrisilviculture (Maize, Barley and Ficus and Grewia), agrihorticulture (Maize, Capsicum, Tomato, Plum and Pear) and silvipasture (Pinus, Acacia and grasses) in Kwaal khad 
watershed in district Solan, Himachal Pradesh. Rajput (2010) reported four agroforestry systems viz, agrihorticulture (Maize, Blackgram, Cauliflower and Apple), agrisilviculture (Maize, Wheat, Grewia and Celtis), agrihorticulture (Maize, Wheat, Tomato, Apple and Grewia) and silvipasture (Grewia, Chir pine, Bauhinia and grasses) in Kullu valley of Himachal Pradesh. Nayak et al., (2011) in Lahaul and Spiti area identified and categorized five different types. These identified agroforestry systems were agri horticulture, agrisilvicultural, agrisilvipastoral, silvipastora and hortipastrol. Major tree species of the area were willow, poplar and apple. A study was carried out by Bijalwan (2012) in mid hill areas of district Tehri Garhwal (Uttarakhand) between the elevation of 1000 to $2000 \mathrm{~m}$ amsl. He reported agrisilviculture (AS), agrihortisilviculture (AHS), agrihorticulture (AH), agrisilvipasture (ASP), silvipasture (SP) and hortisilviculture (HS) systems but reported that the predominant systems were: AS, AHS \& AH. The major trees in AS were: Grewia optiva, Celtis australis, Melia azedarach and Ficus roxburghii etc. along with agricultural crops and under AHS included the combination of Grewia optiva + Malus domestica (apple) + wheat; Quercus leucotrichophora $+M$. domestica (apple) + wheat/potato and Grewia optiva + Prunus domestica (European plum) + barnyard millet etc. The major fruit trees in AH were: $M$. domestica, $P$. domestica and $P$. armeniaca along with routine agricultural crops.

\section{Demographic and socioeconomic status}

\section{Family structure of sampled households}

Family structure represented the total individuals in household comprising adults, children and their respective male-female population in each group. Family structure determines the availability of the family work force on farmer's field. The family structure of the sampled households is shown in Table 2. The perusal of the table shows that the overall mean sex- ratio was 1038 which is higher than the state (Himachal Pradesh) and national averages of 972 and 940 respectively (Census, 2011). It shows that there was no cultural difference on the gender of a child.

The highest sex ratio (1138) was observed in the small category followed by marginal and medium farmer category in the descending order. Adult population constituted 71.47 per cent of the overall population suggesting, thereby, greater availability of the family labour. The average family size was 6.4. Children population constituted $28.53 \%$ of the total population of the sampled household showcasing upcoming family workforce. Similar results regarding family structures were observed by Massingue (2007) who reported an overall sex ratio of 1018 in Naina tikker panchayat of district Sirmaur (HP) which was higher than the state and national averages of 968 and 933 respectively (Census, 2001).

Sharma (2012) has reported higher sex ratio of 988 female to 1000 male in one of the subwatersheds (Sd4e) of Giri Catchment. The average household size of 6.4 is also in line to the findings of Joshi (2011) who reported average family size of 6.5 persons at Karganoo and 7.0 persons at Katli in Rajgarh and Pacchad block of Sirmour (HP). Masoodi (2010) has reported average family size of 5 persons in Solan (HP). Nisha (2013) also reported average family size of 5.53 persons in mid-hills of HP. Sharma et al., (2009) recorded family size of 5.3 to 5.8 persons in Garhwal, Uttarakhand which were at the lower side; Kareemulla et al., (2002) and Mughal et al., (2000) reported an average of 9 persons in a family in Budelkhand region of Uttar Pradesh and in rural Srinagar of Kashmir valley, respectively. 
Table.1 Identification of agroforestry system on the basis of structure and function of the components

\begin{tabular}{|c|c|c|c|c|c|}
\hline \multirow[t]{2}{*}{ Serial No. } & \multirow[t]{2}{*}{ System types } & \multicolumn{3}{|c|}{ No. of families under different categories practicing AF Systems } & \multirow[t]{2}{*}{ Total families } \\
\hline & & Marginal category & Small Category & Medium category & \\
\hline 1 & AS & $4(10.81)$ & $6(13.95)$ & $7(13.21)$ & $17(12.78)$ \\
\hline 2 & ASP & $10(27.03)$ & $12(27.91)$ & $15(28.30)$ & $37(27.82)$ \\
\hline 3 & ASH & $9(24.32)$ & $5(11.63)$ & $7(13.21)$ & $21(15.79)$ \\
\hline 4 & AHS & $2(5.41)$ & $3(6.98)$ & $3(5.65)$ & $8(6.02)$ \\
\hline 5 & HP & - & $2(4.65)$ & $4(7.55)$ & $6(4.51)$ \\
\hline 6 & SP & $12(32.43)$ & $15(34.88)$ & $17(32.08)$ & $44(33.08)$ \\
\hline Total familie & ategory & 37 (100) & 43 (100) & $53(100)$ & $133(100)$ \\
\hline
\end{tabular}

*Figures in the parentheses are percentage to the total.

Table.2 Family structure of sampled households in sub-temperate region of Solan district of Himachal Pradesh

\begin{tabular}{|c|c|c|c|c|c|c|c|c|c|c|}
\hline \multirow[t]{2}{*}{ Farmers' category } & \multirow{2}{*}{$\begin{array}{l}\text { Total no. of } \\
\text { Families }\end{array}$} & \multirow{2}{*}{$\begin{array}{l}\text { Family } \\
\text { Size }\end{array}$} & \multicolumn{3}{|c|}{ Adults } & \multicolumn{3}{|c|}{ Children } & \multirow{2}{*}{$\begin{array}{l}\text { Sex } \\
\text { ratio }\end{array}$} & \multirow{2}{*}{$\begin{array}{l}\text { Average family } \\
\text { members }\end{array}$} \\
\hline & & & Male & Female & Total & Male & Female & Total & & \\
\hline Marginal & 20 & $\begin{array}{c}6.1 \\
(100)\end{array}$ & $\begin{array}{c}2.5 \\
(40.98)\end{array}$ & $\begin{array}{c}2.4 \\
(39.34)\end{array}$ & $\begin{array}{c}4.9 \\
(80.33)\end{array}$ & $\begin{array}{c}0.5 \\
(8.19)\end{array}$ & $\begin{array}{c}0.7 \\
(11.48)\end{array}$ & $\begin{array}{c}1.2 \\
(19.67)\end{array}$ & 1033 & 6.1 \\
\hline Small & 20 & $\begin{array}{c}6.2 \\
(100)\end{array}$ & $\begin{array}{c}2.1 \\
(33.87)\end{array}$ & $\begin{array}{c}2.2 \\
(35.48)\end{array}$ & $\begin{array}{c}4.3 \\
(69.35)\end{array}$ & $\begin{array}{c}0.8 \\
(12.90)\end{array}$ & $\begin{array}{c}1.1 \\
(17.74)\end{array}$ & $\begin{array}{c}1.9 \\
(30.65)\end{array}$ & 1138 & 6.2 \\
\hline Medium & 20 & $\begin{array}{c}6.8 \\
(100)\end{array}$ & $\begin{array}{c}2.2 \\
(32.35)\end{array}$ & $\begin{array}{c}2.25 \\
(33.09)\end{array}$ & $\begin{array}{c}4.45 \\
(65.44)\end{array}$ & $\begin{array}{c}1.3 \\
(19.12)\end{array}$ & $\begin{array}{c}1.05 \\
(15.44)\end{array}$ & $\begin{array}{c}2.35 \\
(34.56)\end{array}$ & 943 & 6.8 \\
\hline Total & 60 & $\begin{array}{c}19.1 \\
(100)\end{array}$ & $\begin{array}{c}\mathbf{6 . 8 0} \\
(35.60)\end{array}$ & $\begin{array}{c}\mathbf{6 . 8 5} \\
(35.86)\end{array}$ & $\begin{array}{c}\mathbf{1 3 . 6 5} \\
(71.47)\end{array}$ & $\begin{array}{c}\mathbf{2 . 6 0} \\
(13.61)\end{array}$ & $\begin{array}{c}2.85 \\
(14.92)\end{array}$ & $\begin{array}{c}\mathbf{5 . 4 5} \\
(28.53)\end{array}$ & 1038 & 6.4 \\
\hline
\end{tabular}

*Figures in the parentheses are percentage to the total 
Table.3 Education status of sampled households in sub-temperate region of Solan district of Himachal Pradesh

\begin{tabular}{|c|c|c|c|c|c|c|c|c|c|c|}
\hline \multirow[t]{2}{*}{ Farmers category } & \multirow[t]{2}{*}{ Primary } & \multirow[t]{2}{*}{ Middle } & \multirow[t]{2}{*}{ Matric } & \multirow[t]{2}{*}{ Secondary } & \multirow[t]{2}{*}{ Graduate } & \multirow[t]{2}{*}{ Illiterate } & \multirow[t]{2}{*}{ Total } & \multirow{2}{*}{$\begin{array}{l}\text { Literacy rate } \\
\qquad \%)\end{array}$} & \multicolumn{2}{|c|}{ Literacy rate \% } \\
\hline & & & & & & & & & Male & Female \\
\hline Marginal & $\begin{array}{c}1.05 \\
(17.21)\end{array}$ & $\begin{array}{c}0.80 \\
(13.11)\end{array}$ & $\begin{array}{c}1.00 \\
(16.39)\end{array}$ & $\begin{array}{c}1.05 \\
(17.21)\end{array}$ & $\begin{array}{c}1.55 \\
(25.41)\end{array}$ & $\begin{array}{r}0.65 \\
(10.66)\end{array}$ & $\begin{array}{c}6.10 \\
(100.00)\end{array}$ & 89.34 & 91.67 & 87.09 \\
\hline Small & $\begin{array}{c}1.25 \\
(20.16)\end{array}$ & $\begin{array}{c}1.00 \\
(16.13)\end{array}$ & $\begin{array}{c}1.20 \\
(19.35)\end{array}$ & $\begin{array}{c}1.10 \\
(17.74)\end{array}$ & $\begin{array}{c}1.05 \\
(16.94)\end{array}$ & $\begin{array}{c}0.60 \\
(9.68)\end{array}$ & $\begin{array}{c}6.20 \\
(100.00)\end{array}$ & 90.32 & 93.1 & 86.36 \\
\hline Medium & $\begin{array}{c}1.50 \\
(22.06)\end{array}$ & $\begin{array}{c}0.85 \\
(12.50)\end{array}$ & $\begin{array}{c}1.65 \\
(24.26)\end{array}$ & $\begin{array}{c}1.40 \\
(20.59)\end{array}$ & $\begin{array}{c}0.90 \\
(13.24)\end{array}$ & $\begin{array}{c}0.50 \\
(7.35)\end{array}$ & $\begin{array}{c}6.80 \\
(100.00)\end{array}$ & 92.65 & 94.29 & 87.88 \\
\hline
\end{tabular}

*Figures in the parentheses are percentage to the total

*The literacy rate is calculated as: (Total literates/total members) x 100

Table.4 Employment status of sampled households

\begin{tabular}{|c|}
\hline Farmers' category \\
\\
\hline Marginal \\
\hline Small \\
\hline Medium \\
\hline Total \\
\hline
\end{tabular}

\begin{tabular}{|c|c|c|c|c|c|c|c|c|c|}
\hline \multirow{2}{*}{$\begin{array}{l}\text { Total no. of } \\
\text { Families }\end{array}$} & \multirow{2}{*}{$\begin{array}{c}\text { Total } \\
\text { members }\end{array}$} & \multicolumn{4}{|c|}{ Government services } & \multicolumn{4}{|c|}{ Industry contract labour } \\
\hline & & M & $\begin{array}{c}\text { Income/ } \\
\text { month (Rs) }\end{array}$ & $\mathbf{F}$ & $\begin{array}{c}\text { Income/ } \\
\text { month (Rs) }\end{array}$ & M & $\begin{array}{c}\text { Income/month } \\
\text { (Rs) }\end{array}$ & $\mathbf{F}$ & $\begin{array}{c}\text { Income/month } \\
\text { (Rs) }\end{array}$ \\
\hline 20 & 122 & 5 & 184000 & 3 & 43800 & 19 & 158500 & 5 & 33000 \\
\hline 20 & 124 & 4 & 136000 & 3 & 10300 & 7 & 58000 & 2 & 27000 \\
\hline 20 & 136 & 5 & 229000 & 2 & 40000 & 9 & 65000 & 2 & 14000 \\
\hline 60 & 382 & 14 & 549000 & 8 & 94100 & 35 & 281500 & 9 & 74000 \\
\hline & & 3.67 & 39214.28 & 2.09 & 11762.50 & 9.16 & 8042.86 & 2.36 & 8222.22 \\
\hline
\end{tabular}


Table.5 Livestock inventory for marginal (a), small (b) and medium (c) category of farmers

\begin{tabular}{|c|c|c|c|c|c|c|c|c|c|c|}
\hline \multirow{2}{*}{$\begin{array}{l}\text { Farmer } \\
\text { category }\end{array}$} & \multirow{2}{*}{$\begin{array}{l}\text { Total no. of } \\
\text { Families }\end{array}$} & \multirow{2}{*}{$\begin{array}{l}\text { Animal } \\
\text { species }\end{array}$} & \multirow{2}{*}{$\begin{array}{c}\text { No. of } \\
\text { animals spp. }\end{array}$} & \multirow{2}{*}{$\begin{array}{l}\text { Average no. per } \\
\text { family }\end{array}$} & \multirow{2}{*}{$\begin{array}{l}\text { Local } \\
\text { breed }\end{array}$} & \multirow{2}{*}{$\begin{array}{l}\text { Improved } \\
\text { breeds }\end{array}$} & \multirow[t]{2}{*}{ Dry } & \multirow[t]{2}{*}{ Milking } & \multicolumn{2}{|c|}{ Young stocks } \\
\hline & & & & & & & & & $\begin{array}{c}\text { No. of } \\
\text { animals spp. }\end{array}$ & $\begin{array}{c}\text { Average no. } \\
\text { per family }\end{array}$ \\
\hline \multirow[t]{4}{*}{$\begin{array}{l}\text { Marginal } \\
\text { (a) }\end{array}$} & \multirow[t]{4}{*}{19} & Cows & $\begin{array}{c}41 \\
(62.12)\end{array}$ & 2.15 & $\begin{array}{c}16 \\
(39.02)\end{array}$ & $\begin{array}{c}25 \\
(60.98)\end{array}$ & $\begin{array}{c}14 \\
(34.15 \\
)\end{array}$ & $\begin{array}{c}24 \\
(58.54)\end{array}$ & $\begin{array}{c}20 \\
(74.07)\end{array}$ & 1.05 \\
\hline & & Buffaloes & $\begin{array}{c}5 \\
(7.57)\end{array}$ & 0.26 & $\begin{array}{c}2 \\
(40)\end{array}$ & $\begin{array}{c}3 \\
(60)\end{array}$ & - & $\begin{array}{c}3 \\
(60)\end{array}$ & $\begin{array}{c}2 \\
(7.41)\end{array}$ & 0.10 \\
\hline & & Bullocks & $\begin{array}{c}16 \\
(24.24)\end{array}$ & 0.84 & $\begin{array}{c}10 \\
(62.5)\end{array}$ & $\begin{array}{c}8 \\
(50)\end{array}$ & - & - & $\begin{array}{c}2 \\
(7.41)\end{array}$ & 0.10 \\
\hline & & Goats & $\begin{array}{c}4 \\
(6.06)\end{array}$ & 0.21 & $\begin{array}{c}4 \\
(100)\end{array}$ & - & - & - & $\begin{array}{c}2 \\
(7.41)\end{array}$ & 0.10 \\
\hline & & Total & $\begin{array}{c}66 \\
(100)\end{array}$ & & & & & & $\begin{array}{c}27 \\
(100)\end{array}$ & \\
\hline \multicolumn{11}{|l|}{5 (b) } \\
\hline \multirow{2}{*}{$\begin{array}{l}\text { Farmer } \\
\text { category }\end{array}$} & \multirow{2}{*}{$\begin{array}{l}\text { Total no. of } \\
\text { Families }\end{array}$} & \multirow{2}{*}{$\begin{array}{l}\text { Animal } \\
\text { species }\end{array}$} & \multirow{2}{*}{$\begin{array}{c}\text { No. of animals } \\
\text { spp. }\end{array}$} & \multirow{2}{*}{$\begin{array}{l}\text { Average no. } \\
\text { per family }\end{array}$} & \multirow{2}{*}{$\begin{array}{l}\text { Local } \\
\text { breed }\end{array}$} & \multirow{2}{*}{$\begin{array}{l}\text { Improved } \\
\text { breeds }\end{array}$} & \multirow[t]{2}{*}{ Dry } & \multirow[t]{2}{*}{ Milking } & \multicolumn{2}{|c|}{ Young stocks } \\
\hline & & & & & & & & & $\begin{array}{c}\text { No. of } \\
\text { animal spp. }\end{array}$ & $\begin{array}{c}\text { Average no. } \\
\text { per family }\end{array}$ \\
\hline \multirow[t]{6}{*}{$\begin{array}{l}\text { Small } \\
\text { (b) }\end{array}$} & \multirow[t]{6}{*}{19} & Cows & $\begin{array}{c}47 \\
(61.84)\end{array}$ & 2.47 & $\begin{array}{l}10.00 \\
(21.28)\end{array}$ & $\begin{array}{c}37 \\
(78.72)\end{array}$ & $\begin{array}{c}12 \\
(25.53)\end{array}$ & $\begin{array}{c}36 \\
(76.60)\end{array}$ & $\begin{array}{c}26 \\
(76.47)\end{array}$ & 1.36 \\
\hline & & Buffaloes & $\begin{array}{c}8 \\
(10.53)\end{array}$ & 0.42 & $\begin{array}{c}5.00 \\
(62.50)\end{array}$ & $\begin{array}{c}3 \\
(37.50)\end{array}$ & $\begin{array}{c}3 \\
(37.50)\end{array}$ & $\begin{array}{c}4 \\
(50.00)\end{array}$ & $\begin{array}{c}3 \\
(8.82)\end{array}$ & 0.16 \\
\hline & & Bullocks & $\begin{array}{c}16 \\
(21.05)\end{array}$ & 0.84 & $\begin{array}{c}5.00 \\
(31.25)\end{array}$ & $\begin{array}{c}11 \\
(68.75)\end{array}$ & - & - & $\begin{array}{c}1 \\
(2.94)\end{array}$ & 0.06 \\
\hline & & Goats & $\begin{array}{c}4 \\
(5.26)\end{array}$ & 0.21 & $\begin{array}{c}4.00 \\
(100.00)\end{array}$ & - & - & - & $\begin{array}{c}4 \\
(11.76)\end{array}$ & 0.21 \\
\hline & & Sheep & $\begin{array}{c}1 \\
(1.32)\end{array}$ & 0.05 & $\begin{array}{c}1.00 \\
(100.00)\end{array}$ & - & - & - & - & - \\
\hline & & Total & $76(100)$ & & & & & & $34(100)$ & \\
\hline
\end{tabular}

*Figures in the parentheses are percentage to the actual owners 
5 (c)

\begin{tabular}{|c|c|c|c|c|c|c|c|c|c|c|}
\hline \multirow{2}{*}{$\begin{array}{l}\text { Farmer } \\
\text { category }\end{array}$} & \multirow{2}{*}{$\begin{array}{l}\text { Total no. of } \\
\text { Families }\end{array}$} & \multirow{2}{*}{$\begin{array}{l}\text { Animal } \\
\text { species }\end{array}$} & \multirow{2}{*}{$\begin{array}{c}\text { No. of } \\
\text { animals spp. }\end{array}$} & \multirow{2}{*}{$\begin{array}{l}\text { Average no. per } \\
\text { family }\end{array}$} & \multirow{2}{*}{$\begin{array}{l}\text { Local } \\
\text { breed }\end{array}$} & \multirow{2}{*}{$\begin{array}{l}\text { Improved } \\
\text { breeds }\end{array}$} & \multirow[t]{2}{*}{ Dry } & \multicolumn{3}{|c|}{ Young stocks } \\
\hline & & & & & & & & Milking & $\begin{array}{l}\text { No. of animals } \\
\text { spp. }\end{array}$ & $\begin{array}{c}\text { Average no. } \\
\text { per family }\end{array}$ \\
\hline \multirow[t]{6}{*}{$\begin{array}{l}\text { Medium } \\
\text { (c) }\end{array}$} & \multirow[t]{6}{*}{18} & Cows & $\begin{array}{c}30 \\
(51.72)\end{array}$ & 1.67 & $\begin{array}{c}6.00 \\
(20.00)\end{array}$ & $\begin{array}{c}24 \\
(80)\end{array}$ & $\begin{array}{c}10 \\
(33.33 \\
)\end{array}$ & $\begin{array}{c}20 \\
(66.67)\end{array}$ & $\begin{array}{c}14 \\
(73.68)\end{array}$ & 0.78 \\
\hline & & Buffaloes & $\begin{array}{c}4 \\
(6.90)\end{array}$ & 0.22 & $\begin{array}{c}1.00 \\
(25.00)\end{array}$ & $\begin{array}{c}3 \\
(75)\end{array}$ & - & $\begin{array}{c}4 \\
(100.00)\end{array}$ & $\begin{array}{c}4 \\
(21.05)\end{array}$ & 0.22 \\
\hline & & Bullocks & $\begin{array}{c}12 \\
(20.69)\end{array}$ & 0.67 & $\begin{array}{c}4.00 \\
(33.33)\end{array}$ & $\begin{array}{c}8 \\
(66.67)\end{array}$ & - & - & - & - \\
\hline & & Goats & $\begin{array}{c}10 \\
(17.24)\end{array}$ & 0.55 & $\begin{array}{c}10.00 \\
(100.00)\end{array}$ & - & - & - & $\begin{array}{c}1 \\
(5.26)\end{array}$ & 0.05 \\
\hline & & Sheeps & $\begin{array}{c}2 \\
(3.45)\end{array}$ & 0.11 & $\begin{array}{c}2.00 \\
(100.00)\end{array}$ & - & - & - & - & - \\
\hline & & Total & $\begin{array}{c}58 \\
(100)\end{array}$ & & & & & & $\begin{array}{c}19 \\
(100)\end{array}$ & \\
\hline
\end{tabular}

*Figures in the parentheses are percentage to the actual owners

Table.6 Land use statistics of farmers of the studied area

\begin{tabular}{|c|c|c|c|c|c|}
\hline \multirow[t]{3}{*}{ Farmers Category } & \multicolumn{3}{|c|}{ Agriculture } & \multirow{3}{*}{$\begin{array}{l}\text { Orchard } \\
\text { (ha) }\end{array}$} & \multirow{3}{*}{$\begin{array}{c}\text { Total area } \\
\text { (ha) }\end{array}$} \\
\hline & \multicolumn{2}{|c|}{ Arable land (ha) } & Non arable land (ha) & & \\
\hline & Irrigated & Non irrigated & Pasture & & \\
\hline Marginal & $\begin{array}{c}0.34 \\
(49.29)\end{array}$ & $\begin{array}{c}0.21 \\
(30.43)\end{array}$ & $\begin{array}{c}0.14 \\
(20.28)\end{array}$ & - & $\begin{array}{c}0.69 \\
(100.00)\end{array}$ \\
\hline Small & $\begin{array}{c}0.47 \\
(36.43)\end{array}$ & $\begin{array}{c}0.20 \\
(15.50)\end{array}$ & $\begin{array}{c}0.58 \\
(44.96)\end{array}$ & $\begin{array}{c}0.04 \\
(3.10)\end{array}$ & $\begin{array}{c}1.29 \\
(100.00)\end{array}$ \\
\hline Medium & $\begin{array}{c}0.82 \\
(24.62)\end{array}$ & $\begin{array}{c}0.97 \\
(29.14)\end{array}$ & $\begin{array}{c}1.38 \\
(41.44)\end{array}$ & $\begin{array}{c}0.16 \\
(4.80)\end{array}$ & $\begin{array}{c}3.33 \\
(100.00)\end{array}$ \\
\hline Total & $\begin{array}{c}1.63 \\
(24.55)\end{array}$ & $\begin{array}{c}1.38 \\
(29.13)\end{array}$ & $\begin{array}{c}2.1 \\
(41.47)\end{array}$ & $\begin{array}{c}\mathbf{0 . 2 0} \\
(4.85)\end{array}$ & $\begin{array}{c}\mathbf{5 . 3 1} \\
(100.00)\end{array}$ \\
\hline
\end{tabular}




\section{Educational status of sampled households}

Table 3 shows the educational status of the sampled households. It was found necessary to study educational status as it determines the farmer's awareness level thereby influencing the adoption mechanism of suggested modern technologies and rational decision making pertaining to adoption of different agroforestry systems. The literacy rate varied from 89.34 to 92.65 per cent among different farmer categories. The result revealed that the literacy rate was recorded highest in medium farmer (92.65) followed by small (90.32) and marginal farmer (89.34) category respectively. The males were found to be more literate in comparison to females in all the categories of farmers in the study area. Many workers who have worked on 'socioeconomic status' in other parts of Himachal Pradesh reported that literacy percent varied from $75.79 \%$ in Katli panchayat and to $88.09 \%$ in Karganoo panchayat in Rajgarh and Sarah block of district Sirmour (Himachal Pradesh), (Joshi, 2011). While, Nisha (2013) reported overall level of literate persons in a family as $92.23 \%$ in mid-hills of Sirmour district. Likewise, in other parts of India, Sharma et al., (2009) recorded $80 \%$ literacy in villages of Garhwal, Uttarakhand and Mughal et al., (2000) reported $31 \%$ literacy in rural Srinagar of Kashmir valley.

\section{Employment status of sampled households}

There were four different employment avenues in the study area (Table 4). The sampled farmers met their livelihood through government jobs, industrial labour, selfemployment and farm labour. Males were dominating in all the three income generating avenues except in the farm labour. With regard to government jobs, it was found that 3.67 per cent of the males were engaged in this sector as against 2.09 per cent of females.
Similar trend was observed in case of industrial labour and self-employment opportunities indicating higher percentage of males employed as against the females. This clearly points out that towards increased opportunities of cash generations being available to the male counterpart in comparison to females. However, the farm activities were dominated by the females. It was observed that 22.25 per cent males were fully engaged in the farm activities as against 24.61 per cent of females. Men were found to be more interested in off farm cash generating activities, whereas, females were dominating in the household and own farm activities. The average income per month for government job was found to be Rs. 39214.28 to males and Rs. 11762.50 for females. Selfemployment was also playing an important role in income generation. Females were mostly engaged in non-income generating activities.

\section{Livestock inventory of the sampled households}

The status of livestock in the sampled farmers is shown by Table 5a, 5b and 5c. Livestock was found to play a crucial role in the farming systems of the sampled households. The Tree/crop -livestock systems provide green and dry fodder and in return gives manure to the crops. Livestock is a source of income to the farmers in terms of milk, wool as well as meet. Perusal of the data from Table 5a revealed that cow was most preferred domesticated animal with an average value (2.15) followed by other species of animal's viz. Buffaloes and goats in descending order. Also, in small category cow was the major domesticated species followed by other species of livestock. Similar trends were also observed in medium category of farmers where cows showed the highest value (1.7) followed by other livestock species. The data indicates that the all farmer categories have 
diversified systems. It was also evident from the table that cows were found to be the major livestock species reared for the purpose of milk followed by the buffalo. It was also observed that number of bullocks per family were less as it was due to reason that land preparation was done by owned as well as by hired bullocks in addition to tractors. All the sampled farmers in the study area preferred more of the improved breeds than the local ones among all animal species which can be ascribed to the reason that improved breeds have more productivity and thus leading to higher economic gains. No poultry, pisiculture, apiculture and other accessory systems were observed to be followed by the farmers of the study area. Thus, one can draw conclusion that livestock was playing an important role in farm sector activities to augment the overall income of the farmers.

\section{Land use statistics of sampled households}

Different land use statistics among the sampled farmers in the study area is depicted in the Table 6. Average area under arable land constitutes 53.68 per cent combining both irrigated and unirrigated areas out of which major portion of arable land was dependent on rainfall. Besides, considerable amount of arable land was also under irrigation facilities. All farmers in the sampled areas do practise horticulture but in lesser proposition of their lands with total of only 4.85 per cent. With regard to pasture land, it was observed that medium farmers have higher area under it and the lowest was in the marginal category. No woodlots were observed in the study area as people were meeting their fuel, fodder and other needs through trees retained on agriculture and pasture land. Similar survey of agroforestry systems in Balh valley of Mandi district (Himachal Pradesh) by Upadhayaya (1997) revealed that 97 per cent of the household were in marginal and small categories covering about 89 per cent of the total land area. Further, he reported that 83 per cent of land area was under agriculture and cultivation done under rainfed condition which was in contrary to our present findings.

\section{Acknowledgement}

Author is thankful to Head of the Department of Silviculture and Agroforestry, College of Forestry for providing necessary facility and also to the farmers for their co-operation to carry out this research work.

In conclusion, it is worth mentioning here that the overall family sex ratio was reported to be 1038, which was higher than the state and national average of 972 and 940 respectively depicting no cultural difference on the gender of a child. Literacy rate varied from 89.34 to $92.65 \%$ among different categories of farmers. Males were dominating in all the income generation avenues except in the farm labor. Improved breeds of livestock was preferred more compared to local ones in all animal species which can be ascribed that improved breeds have more productivity and thus leading to higher economic gains. Average area under arable land constitutes $53.68 \%$ combining both irrigated and un irrigated areas out of which major portion of arable land was dependent on rainfall. All farmers in the sampled areas do practice horticulture but in lesser proposition of their lands with a meagre total of only 4.85 per cent. With regard to pasture land, it was observed that medium farmers have higher area under it and the lowest being in the marginal category. No woodlots were observed in the study area as people were meeting their fuel, fodder and other needs through trees retained on agriculture and pasture land. Agroforestry systems observed in the studied area helped farmers to increase their farm income, easy availability of firewood and timber on the farmland. Finally, it can be concluded that agroforestry is 
prerequisite for the conservation of natural forest and socio-economic upliftment of rural population.

\section{References}

Al-assaf, A., Nawash, O and Omari, M. 2014. Identifying forest ecosystem services through socio-ecological bundles: a case study from northern Jordan. International Journal of Sustainable Development \& World Ecology 21(4): 314-321.

Bijalwan, A. 2012. Land-use and vegetation pattern in traditional agroforestry systems in mid hills of Garhwal Himalaya. Journal of Progressive Agriculture 3(2): 6-13

Goswami, S. 2009. Appraisal of agroforestry land use system for their carbon sequestration potential. M.Sc. Thesis. Dr. Y.S. Parmar University of Horticulture and Forestry, Nauni- Solan (H.P.), India.134p.

Hossain, Md Sarwar, Eigenbrod, Felix, Johnson, Fiifi Amoako, and Dearing, John A. (2016): Unravelling the interrelationships between ecosystem services and human wellbeing in the Bangladesh delta, International Journal of Sustainable Development \& World Ecology: 1-15.

Joshi, R. 2011. Studies on the vulnerability of agroforestry resources to climate change and its impact on farmers' economy in mid hills of western Himalayas. Ph.D. Thesis. Dr. Y. S. Parmar University of Horticulture and Forestry, Nauni, Solan (H.P.), India. 134p.

Kachru, S. R. 1997. Diagnostic survey and productivity appraisal of agroforestry systems in sub temperate and sub humid region of Himachal Pradesh. M.Sc. Thesis. Dr. Y. S. Parmar University of Horticulture and Forestry, Nauni, Solan (H.P.), India.162p.
Kareemula, K., Rizvi, R. H., Singh, R., and Dwevedi. 2002. Trees in rainfed agroecosystem, socio- economic investigation in Bundelkhand region. Indian Journal of Agroforestry. 4(1): 53-56.

Kumari, A., Sehgal, R. N., and Kumar, S. 2008. Tradition agroforestry system practiced in (Lahaul and Spiti) and Kinnaur districts of Himachal Pradesh. Indian forester 43(2): 1003-1009

Masoodi, H. R. 2010. Vegetation dynamics and land use cover of 'Ga3a' microwatershed of Giririver in Solan district of Himachal Pradesh, India. M.Sc. Thesis, Dr. Y.S. Parmar University of Horticulture and Forestry, Nauni-Solan (H.P.) India. 120p.

Massingue, E. J. 2007. Structure and functions of different agroforestry systems in subtemperate region of Himachal Pradesh, India. M.Sc. Thesis. Dr. Y. S. Parmar University of Horticulture and Forestry, Nauni, Solan (H.P.), India. 91p.

Mercer, D.E.1993 A framework for analyzing the socioeconomic impacts of agroforestry projects. Southeastern Center for Forest Economics Research, Research Triangle Park, NC. FPEI Working Paper No. 52, 34 pp

Mughal, A. H., Ara, T., and Bhattacharya, P. 2000. Socioeconomic aspects of agroforestry in rural Srinagar of Kashmir valley. Indian forester 126 (8): 234-24

Nayak, P. D., Bawa, R., and Gupta, T. 2011. Agroforestry Systems of Lahaul and Spiti District of Himachal Pradesh, Western Himalaya. Indian Journal of Ecology 38: 129-131

Nisha, D. 2013. Ameliorating farm income through agroforestry practices in midhill zone of Himachal Pradesh. M.Sc. Thesis. Dr. Y. S. Parmar University of 
Horticulture and Forestry, Nauni, Solan (H.P.). 94p.

Rajput, B. S. 2010. Bio-economic appraisal and carbon sequestration potential of different land use systems in temperate north-western Himalayas. Ph.d Thesis. Dr. Y S Parmar University of Horticulure and Forestry, Nauni, Solan (H.P.), India. 95p.

Seabrook, L., McAlpine, C., and Fensham, R. J. 2008. What influences farmers to keep trees? A case study from the Brigalow Belt Queensland Australia. Landscape Urb. Plan. 84: 266-281.

Sharma, B. M., Gupta, G. P., Rathore, S. S., and Prasad, R. N. 1993. Evaluation of silvipastoral systems under arid condition in Rajasthan. Indian Journal of Agriculture Economics 46(3): 422427

Sharma, C. M., Gairola, S., Suya, S., and Ghildiyal, S. K. 2009. Forest resources use patterns in socioeconomic status. Mountain Research and Development 29(5): 119-128.

Sharma. 2012. GIS based land use planning for watershed management. Ph.D. Thesis. Dr. Y.S. Parmar University of Horticulture and Forestry, Nauni-Solan (H.P.), India. 155p.

Upadhyaya, K. 1997. Diagnostic survey of agroforestry system in Balh valley of Himachal Pradesh. M.Sc. Thesis. Dr. Y. S. Parmar University of Horticulture and Forestry, Nauni Solan (H.P.), India. $141 \mathrm{p}$.

\section{How to cite this article:}

Pradeep Kumar, C.L. Thakur, Pradeepen Rai and Kaushal Attri. 2018. Identification of Existing Agroforestry Systems and Socio-Economic Assessment in Kandaghat Block of Solan District, Himachal Pradesh, India. Int.J.Curr.Microbiol.App.Sci. 7(04): 3815-3826. doi: https://doi.org/10.20546/ijcmas.2018.704.429 